\section{Avaliação da adesão ao tratamento imunossupressor em pacientes transplantados renais da cidade de Cruz Alta-RS}

\author{
Evaluation of accession to immunosuppressive treatment in renal \\ transplanted patients of the city of Cruz Alta-RS
}

RESUMO

Thiago de Souza Claudino e Adriele Lacerda

Objetivo: Avaliar a adesão ao tratamento imunossupressor de pacientes atendidos em uma unidade de terapia renal. Métodos: Trata-se de um estudo descritivo, prospectivo e transversal, com pacientes de ambos os sexos, com idades iguais ou acima de 18 anos, alfabetizados ou com cuidador e familiar responsável, transplantados renais e que ainda fazem uso de terapia imunossupressora e que aceitaram participar do estudo, após assinatura do Termo de Consentimento Livre e Esclarecido. Os dados referentes à pesquisa foram coletados entre os meses de agosto a outubro de 2017, através da análise dos prontuários, bem como aplicou-se uma ficha clínica aos pacientes e, posteriormente, para avaliar a adesão ao tratamento, foram utilizados os instrumentos Brief Medication Questionnaire (BMQ) e Morisky-Green Test (TMG). Resultados: Participaram deste estudo dez pacientes ( $n=10)$, com 23 a 77 anos, sendo $60 \%$ do sexo feminino. Observou-se nos pacientes que há relação entre hipertensão arterial, Diabetes Mellitus e progressão da Doença Renal Crônica. Os resultados demonstraram que nos domínios Regime, Recordação e Crença do questionário BMQ, que avaliou as opiniões dos pacientes relacionadas à eficácia do tratamento e efeitos colaterais, além da administração dos medicamentos, observou-se que $40 \%$ dos pacientes foram aderentes, $30 \%$ prováveis a baixa adesão e $30 \%$ baixa adesão. No domínio regime, $70 \%$ dos pacientes obtiveram alta adesão, $20 \%$ provável adesão e $10 \%$ provável baixa adesão, identificando que a maioria dos pacientes aceitaram bem a terapia medicamentosa. No TMG, que avaliou o uso habitual de medicamentos, notou-se que, $70 \%$ dos pacientes obtiveram alta adesão, $20 \%$ provável adesão e 10\% provável baixa adesão. Considerações finais: Através do estudo, conclui-se que a maioria dos pacientes aceitou bem a terapia, porém as maiores dificuldades são relacionadas à eficácia e ao cumprimento de horários de administração dos medicamentos.

PALAVRAS-CHAVE: Insuficiência Renal Crônica; Imunossupressores; Cooperação e Adesão ao Tratamento.

\section{ABSTRACT}

Objective: To assess adherence to immunosuppressive treatment of patients seen at a renal therapy unit. Methods: This is a descriptive, prospective and cross-sectional study, with patients of both sexes, aged 18 years or over, literate or with a responsible caregiver and family member, kidney transplant patients who still use immunosuppressive therapy and who agreed to participate in the study, after signing the Informed Consent Form. The data referring to the research were collected between the months of August and October 2017, through the analysis of the medical records, as well as a clinical record was applied to the patients and, subsequently, to assess adherence to treatment, the Brief Medication instruments were used Questionnaire (BMQ) and Morisky-Green Test (TMG). Results: Ten patients $(n=10)$, aged 23 to 77 years, participated in this study, $60 \%$ of whom were female. It was observed in patients that there is a relationship between arterial hypertension, Diabetes Mellitus and progression of Chronic Kidney Disease. The results showed that in the Regime, Recall and Belief domains of the $B M Q$ questionnaire, which evaluated the patients' perceptions related to the effectiveness of the treatment and effects, in addition to the administration of the drugs, it was observed that $40 \%$ of the patients were adherent, $30 \%$ likely low adherence and $30 \%$ low adherence. No domain regimen, $70 \%$ of patients achieved high adherence, $20 \%$ probable adherence and $10 \%$ probable low adherence, identifying that most patients accepted drug therapy well. In the TMG, which assessed the usual use of medications, it was noted that $70 \%$ of patients achieved high adherence, $20 \%$ likely adherence and 10\% likely low adherence. Final considerations: Through the study, it was concluded that the majority of patients accepted the therapy well, but as major difficulties are related to the effectiveness and compliance with medication administration schedules.

KEYWORDS: Renal Insufficiency, Chronic; Immunosupressives Agents; Treatment Adherence and Compliance. 


\section{INTRODUÇÃO}

Os rins são órgãos pares que fazem parte do sistema urinário, situando-se junto à parede posterior fora da cavidade peritoneal, a cada lado da coluna vertebral. São fundamentais na regulação da homeostase corpórea e na eliminação de produtos do metabolismo orgânico, como ureia, creatinina e ácido úrico, além de controlar as concentrações da água e da maioria dos constituintes dos líquidos do organismo. Disfunções em algum destes processos podem ser observados pela diminuição da taxa de filtração glomerular, resultando no surgimento da Doença Renal Crônica (DRC), que, quando associada a grupos de risco (hipertensos, diabéticos, idosos, pacientes com doenças cardiovasculares, histórico familiar e que utilizam medicamentos nefrotóxicos), pode agravar o quadro clínico do paciente, elevando os índices de transplantes renais e tornando-se assim um problema de saúde pública mundial. ${ }^{1,2}$

Em paciente com DRC em fase avançada, o transplante renal é a terapia mais indicada, sendo o tratamento imunossupressor o esquema terapêutico de escolha. O grande progresso no desenvolvimento destes fármacos, desde 0 inicio da realização de transplantes renais na década de 60, tem por objetivo estabelecer o estado de imunossupressão, prevenindo assim o desenvolvimento de episódios de rejeição aguda e crônica., ${ }^{3,4}$

As doses e esquemas de terapia são muito variáveis, devendo ser ajustada e monitorada por toda a vida do transplantado, a fim de garantir o funcionamento do enxerto. Porém, apesar da importância da utilização destes fármacos para resultados positivos na vida do paciente transplantado renal, nota-se que em longo prazo estes pacientes demonstram complicações, que implicam na dificuldade a adaptação à terapia, resultando em falhas. 0 conceito de adesão medicamentosa caracteriza-se como um processo no qual os sujeitos envolvidos são influenciados por vários fatores que determinam a sua continuidade ou descontinuidade, afetando principalmente a qualidade de vida. $3,5,6$

Assim, considerando que o atual tratamento de escolha no pós-transplante renal é a terapia imunossupressora de uso contínuo, com o uso concomitante de outros medicamentos, e que diversos fatores podem determinar a qualidade do tratamento, torna-se necessário avaliar a adesão ao tratamento. 0 presente estudo tem por objetivo avaliar a adesão ao tratamento imunossupressor de pacientes atendidos em uma unidade de terapia renal, através da análise de prontuários e consequentemente aplicação dos questionários Brief Medication Questionnaire (BMQ) e Morisky-Green Test (TMG).

\section{MÉTODO}

O presente trabalho trata-se de um estudo descritivo, prospectivo e transversal. A pesquisa foi realizada a domicílio com os pacientes atendidos em uma unidade de terapia renal na cidade de Cruz Alta, Rio Grande do Sul, Brasil, anexada juntamente a um hospital e referência no serviço oferecido.

Como critério de inclusão, foram selecionados pacientes de ambos os sexos, com idades iguais ou acima de 
18 anos, alfabetizados ou com cuidador e familiar responsável, transplantados renais e que ainda fazem uso de terapia imunossupressora e que aceitaram participar do estudo, após assinatura do Termo de Consentimento Livre e Esclarecido, lido e assinado por todos os pacientes participantes do estudo ou responsáveis.

Os dados referentes à pesquisa foram coletados entre os meses de agosto a outubro de 2017, através da análise dos prontuários, para verificação de história clínica e de esquema de terapia dos pacientes selecionados, bem como aplicou-se uma ficha clínica aos pacientes, onde se pesquisou variáveis como peso, altura, circunferência abdominal, Índice de Massa Corpórea (IMC) (Índice de Massa Corpórea), data de transplante, renda média salarial, grau de instrução e a situação de saúde do paciente em estudo. Posteriormente, para avaliar a adesão ao tratamento, foram utilizados os instrumentos BMQ e TMG. ${ }^{7}$

O BMQ foi avaliado através da pontuação dos domínios Crença, Regime e Recordação, classificando como alta adesão se nenhuma resposta positiva em algum domínio, provável adesão se resposta positiva em um domínio, provável baixa adesão se resposta positiva em dois domínios e baixa adesão se resposta positiva em três domínios. 0 instrumento também avaliou o domínio regime separadamente, classificando como alta adesão se nenhuma resposta positiva, provável adesão se uma resposta positiva, provável baixa adesão se duas respostas positivas e baixa adesão se três respostas positivas.

O TMG avaliou o comportamento do paciente frente ao uso continuo de medicamentos imunossupressores, através de quatro perguntas. A classificação foi realizada conforme as respostas das questões, sendo considerado um paciente com alto grau de adesão se nenhuma resposta fosse positiva, provável adesão se uma a duas respostas fossem positivas, provável baixa adesão se três respostas fossem positivas e e acima disso considerou-se como baixa adesão.

A amostragem foi constituída de uma população de dez pacientes $(n=10)$, de uma lista de vinte e cinco atendidos na unidade. As variáveis foram apresentadas em média, desvio padrão e porcentagem.

Quanto aos aspectos éticos, o projeto de pesquisa foi elaborado conforme a legislação sobre pesquisas envolvendo seres humanos, protocolado e aprovado pelo Comitê de Ética em Pesquisa (CEP) da Universidade de Cruz Alta - UNICRUZ, sob Parecer n² 2.078.603 e CAAE nº 66814917.0000.5322.

\section{RESULTADOS E DISCUSSÃO}

Dos pacientes atendidos na Unidade de Terapia Renal da Cidade de Cruz Alta-RS, foram contatados vinte e cinco pacientes, $40 \%$ da população-alvo, devido ao fato de que alguns pacientes não são atendidos com tanta frequência, havendo desatualização dos dados cadastrais. Dos 25 pacientes contatados, 10 pacientes aceitaram participar da pesquisa. Os resultados do estudo relacionados a variáveis sociais, conforme ficha clínica aplicada aos pacientes, estão 
demonstrados na Tabela 1, representando um grupo com idades médias entre 23 a 77 anos, sendo $60 \%$ do sexo feminino. Em um estudo realizado em um hospital no município de Porto Alegre (RS), com pacientes renais crônicos submetidos à hemodiálise, houve predominância de pacientes do sexo masculino (67,5\%), sendo $60 \%$ dos pacientes com mais de 60 anos.8

Com relação ao grau de instrução o estudo demonstrou uma população com nível de instrução bem distribuído, com isso pode-se observar que os pacientes com maior escolaridade demonstravam maior facilidade em relatar sobre a terapia, maior controle sobre horários e posologias dos medicamentos imunossupressores. A renda média salarial dos participantes foi entre 1 a 3 salários mínimos, o que muitas vezes, segundo os entrevistados, dificulta a compra de medicamentos para outras enfermidades, já que a maioria dos pacientes fazem uso de polimedicação e muitas vezes não conseguem obtê-las na rede pública. Neste contexto, $70 \%$ dos pacientes relataram não conseguir comprar todos os medicamentos para uso contínuo, embora $100 \%$ consigam os medicamentos imunossupressores gratuitamente, sem atrasos na entrega.

Tabela 1: Distribuição dos pacientes Transplantados renais, conforme variáveis sociais, Cruz Alta- RS, 2017.

\begin{tabular}{ccc}
\hline Variáveis & $\mathbf{n}$ & $\%$ \\
\hline Faixa etária & 4 & $40 \%$ \\
23 a 40 anos & 3 & $30 \%$ \\
41 a 60 anos & 3 & $30 \%$ \\
61 a 77 anos & & \\
Sexo & 4 & $40 \%$ \\
Masculino & 4 & $60 \%$ \\
Feminino & 6 & $30 \%$ \\
Grau de instrução & & $20 \%$ \\
Fund. Incompleto & 3 & $10 \%$ \\
Fund. Completo & 2 & $20 \%$ \\
Méd. Incompleto & 1 & $20 \%$ \\
Méd. Completo & 2 & \\
Superior. Completo & 2 & $50 \%$ \\
Renda Individual & 2 & $50 \%$ \\
1 a 2 s.m & & \\
2 a 3 s.m & 5 & \\
Medicações imunossupressoras gratuitas & 5 & \\
Sim & & \\
\hline
\end{tabular}


Não

Condições de comprar demais med.

\begin{tabular}{lll} 
Sim & 3 & $30 \%$ \\
Não & 7 & $70 \%$ \\
\hline
\end{tabular}

Segundo a Tabela 2, quando avaliados a composição corporal, 40\% dos pacientes obtiveram IMC entre 18,5 a 25 , sendo considerados com o peso ideal, outros $40 \%$ obtiveram IMC $\geq 25<30$, sendo considerados com sobrepeso, e $20 \%$ com IMC $\geq 30$, caracterizando como obesidade de grau I. ${ }^{9}$

Observa-se também na Tabela 2 que 100\% das mulheres em estudo apresentaram circunferência abdominal acima do ideal, sendo superior aos valores encontrados na população masculina, que apresentou $75 \%$ de circunferência acima do ideal. Estes resultados mostraram- se semelhantes àqueles observados em um estudo que avaliou a circunferência abdominal em mulheres hipertensas, relacionando a prevalência de doenças metabólicas e cardiovasculares com 0 aumento da circunferência abdominal. ${ }^{9}$

Tabela 2: Distribuição dos pacientes transplantados de acordo com composição corporal. Cruz Alta- RS, 2017.

\begin{tabular}{ccc}
\hline Variáveis & n & $\%$ \\
\hline IMC & & \\
18,5 a 25 & 4 & $40 \%$ \\
$\geq 25<30$ & 4 & $40 \%$ \\
$\geq 30$ & 2 & $20 \%$ \\
Peso & & \\
52 a 58 kg & 2 & $20 \%$ \\
58,5 a $70 \mathrm{~kg}$ & 4 & $40 \%$ \\
70,5 a $85 \mathrm{~kg}$ & 4 & $40 \%$
\end{tabular}

Altura

$1,53 \mathrm{~m}$ a $1,63 \mathrm{~m}$

7

$70 \%$

$1,64 \mathrm{~m} \mathrm{a} 1,76 \mathrm{~m}$

3

$30 \%$

\section{Circunf. Abdominal}

Masculino

$\leq 94 \mathrm{~cm}$

1

$25 \%$

$94 \mathrm{a} 102 \mathrm{~cm}$

5

$50 \%$

$\geq 102 \mathrm{~cm}$

1

$25 \%$

Feminino 


\begin{tabular}{ccc}
$\leq 80 \mathrm{~cm}$ & 0 & $0 \%$ \\
$80 \mathrm{a} 88 \mathrm{~cm}$ & 1 & $10 \%$ \\
$\geq 88 \mathrm{~cm}$ & 6 & $90 \%$ \\
\hline
\end{tabular}

A distribuição dos pacientes de acordo com as complicações de saúde são observados na tabela 3, onde observase que $90 \%$ dos participantes tiveram hipertensão arterial pré-transplante e $50 \%$ ainda fazem tratamento para controle. 0 presente estudo também demonstrou que $20 \%$ dos pacientes realizou transplante duas vezes, devido à rejeição aguda do enxerto, estando relacionadas ao aumento da hipertensão arterial, não adaptação a terapia inicial e ocorrências de infecções. Além disso, nota-se que $70 \%$ dos participantes relataram histórico familiar de hipertensão arterial e $20 \%$ desenvolveram diabetes melittus pré-transplante, seguindo com tratamento atualmente. Estes resultados confirmam a relação entre hipertensão arterial, diabetes mellitus e a progressão da doença renal crônica e consequentemente 0 transplante renal. ${ }^{1,3}$ Em estudo realizado em Porto Alegre-RS, hipertensão arterial e diabetes também foram relatados por 85 e $42,5 \%$ dos pacientes, respectivamente. ${ }^{8}$

Tabela 3: Distribuição dos pacientes transplantados renais de acordo com as complicações de saúde. Cruz Alta- RS, 2017.

\begin{tabular}{ccc}
\hline Variáveis & $\mathrm{n}$ & $\%$ \\
\hline Complicações & 8 & $80 \%$ \\
Transplantado uma vez & 2 & $20 \%$ \\
Transplantado duas vezes & & \\
H.A pré transplante & 9 & $90 \%$ \\
Sim & 1 & $10 \%$ \\
Não & & \\
H.A pós transplante & 5 & $50 \%$ \\
Sim & 5 & $50 \%$ \\
Não & & \\
Histórico H.A familiar & 7 & $70 \%$ \\
Sim & 3 & $30 \%$ \\
Não & & \\
D.M pré transplante & 2 & $20 \%$ \\
Sim & 8 & \\
Não & & \\
D.M pós transplante & 2 & \\
\end{tabular}


Sim

Não

Histórico D.M familiar

Sim

Não
$80 \%$

$70 \%$

$30 \%$

Com relação à adesão ao tratamento, foi utilizado o questionário Brief Medication Questionnaire (BMQ), que foi validado em pacientes hipertensos nos Estados Unidos da América (EUA), e utilizado no Brasil em estudos com pacientes hipertensos atendidos em Unidades de Saúde, em Porto Alegre (RS) e em Curitiba (PR) ${ }^{10,11}$. Estes estudos propõem identificar as barreiras de adesão, através da avaliação do tratamento, recordação e crenças do paciente relacionadas à terapia medicamentosa, para isto questionário foi dividido em três domínios.

Quando avaliados os três domínios (regime, recordação e crenças) (Tabela 4), pode-se observar que $40 \%$ dos pacientes eram prováveis aderentes à terapia, pois obtiveram resposta positiva em um domínio, 30\% dos pacientes obtiveram provável baixa aderência ao tratamento, por terem respondido respostas positivas em dois domínios, e 30\% representaram baixa adesão à terapia, pois obtiveram resposta positiva em três domínios. Nesta avaliação, nenhum paciente obteve alta adesão, e isto se deve principalmente as respostas positivas nos regimes de crenças, que buscou avaliar as opiniões dos pacientes relacionadas à eficácia do tratamento e efeitos colaterais, e no regime recordação, onde avaliou-se sobre a administração dos medicamentos, perguntando da dificuldade de lembrar de tomar o medicamento e se o paciente faz terapia com posologia mais de uma vez ao dia. Os resultados foram semelhantes aos encontrados em pacientes renais crônicos de Porto Alegre-RS, em que, no BMQ, nenhum paciente foi classificado como alta adesão ou provável alta adesão, sendo que $35 \%$ foram classificados como provável baixa adesão, enquanto a maioria (65\%) foi classificada como baixa adesão. ${ }^{8}$ Os resultados do estudo são inferiores ao estudo que avaliou a utilização do instrumento com pacientes hipertensos em Curitiba-PR, que obteve em média de $59 \%$ de pacientes com baixa adesão. ${ }^{10}$

Quando avaliado somente domínio regime, observou-se a reação do paciente frente à terapia prescrita, através de cinco perguntas relacionadas à listagem dos medicamentos tomados na última semana, quantos dias ingeriu, quantas vezes ao dia, quantos comprimidos, quantas vezes houve o esquecimento e como o medicamento funciona. Observouse que $70 \%$ dos pacientes obtiveram alta adesão, $20 \%$ provável adesão e 10\% provável baixa adesão, identificando que a maioria dos pacientes aceitaram bem a terapia medicamentosa.

Diferente do estudo com pacientes hipertensos em Porto Alegre- RS, onde nenhum paciente obteve alta adesão. ${ }^{11}$

Tabela 4: Distribuição dos participantes de acordo com as variáveis a adesão ao tratamento imunossupressor, segundo 
BMQ. Cruz Alta-RS, 2017.

\begin{tabular}{lll}
\hline \multicolumn{1}{c}{ Variáveis } & $\mathbf{n}$ & $\%$ \\
\hline \multicolumn{1}{c}{ BMQ } & & \\
Alta adesão (nenhuma resposta positiva) & 0 & $0 \%$ \\
Provável adesão (Resposta positiva em 1 domínio) & 4 & $40 \%$ \\
Provável baixa adesão (Resposta positiva em 2 domínios) & 3 & $30 \%$ \\
Baixa adesão (Resposta positiva em 3 domínios) & 3 & $30 \%$ \\
$\quad$ BMQ domínio regime & & \\
Alta adesão (nenhuma resposta positiva) & 7 & $70 \%$ \\
Provável adesão (uma resposta positiva) & 2 & $20 \%$ \\
Provável baixa adesão (2 respostas positivas) & 1 & $10 \%$ \\
Baixa adesão (ะ3 respostas positivas) & 0 & $0 \%$ \\
\hline
\end{tabular}

Adistribuição dos participantes de acordo com as variáveis relacionadas à adesão ao tratamento imunossupressor, segundo escore do TMG, são observadas na tabela 5, que propôs uma avaliação aos pacientes frente ao uso habitual dos medicamentos prescritos. No estudo, verificou-se que $70 \%$ dos pacientes obtiveram alta adesão medicamentosa, pois não responderam nenhuma resposta positiva, 20\% provável adesão, por terem respondido pelo menos uma resposta positiva, e 10\% provável baixa adesão, devido a duas respostas positivas. Em pacientes renais crônicos submetidos à hemodiálise, realizado em Porto Alegre-RS, o TMG revelou moderada adesão (57,5\%), alta adesão (32,5\%) e baixa adesão (10\%), sendo este último resultado semelhante ao observado no presente estudo, mas que teve maior porcentagem para alta adesão..$^{8}$ O resultado do presente estudo se mostrou diferente do estudo que avaliou os pacientes hipertensos em Porto Alegre (RS), onde nenhum paciente obteve alta adesão, sendo a maioria classificada entre moderada a baixa adesão. ${ }^{11}$

Tabela 5: Distribuição dos participantes de acordo com as variáveis a adesão ao tratamento imunossupressor, segundo escore do TMG. Cruz Alta-RS, 2017.

\begin{tabular}{ccc}
\hline Variáveis & $\mathbf{n}$ & $\%$ \\
\hline Questão $\mathbf{n}^{\mathbf{0}}$ & & \\
Sim & 1 & $10 \%$ \\
Não & 10 & $100 \%$ \\
Questão $\mathbf{n}^{\mathbf{0} 2}$ & & \\
Sim & 3 & $30 \%$ \\
Não & 7 & $70 \%$
\end{tabular}




\section{Questão ${ }^{03}$}

$\begin{array}{lll}\text { Sim } & 1 & 10 \% \\ \text { Não } & 9 & 90 \%\end{array}$

\section{Questão n ${ }^{04}$}

Sim

Não

\section{Escore TMG*}

Alta adesão (nenhuma resposta positiva)

Provável adesão (1 resposta positiva)

Provável baixa adesão (2 respostas positivas)

Baixa adesão ( $\geq 3$ respostas positivas)
1

9

$10 \%$

$90 \%$

$70 \%$

$20 \%$

$10 \%$

$0 \%$

Além do $B M Q$ e do TMG, outros questionários vem sendo empregados com a finalidade de avaliar a adesão ao tratamento em pacientes com DRC. Em duas cidades do Rio de Janeiro (Itaboraí e Niterói), aplicou-se o Questionário de avaliação sobre a adesão do portador de doença renal crônica em hemodiálise (QADRC-HD), em que o domínio medicação foi o que apresentou maior percentual de pacientes aderentes $(93,6 \%)$, seguindo de dieta $(85,9 \%)$, ingestão hídrica $(82,1 \%)$ e hemodiálise (68\%). ${ }^{12}$ Empregando-se a escala BAASIS (Basel Assessment of Adherence with immunosuppressive medication Scale), em pacientes de um ambulatório pós-transplante de São Paulo-SP, os resultados isolados dos itens avaliados pela BAASIS $®$ apontaram uma adesão de 94,5\%, embora o escore geral mostrou uma não-adesão de $41,4 \%$ dos pacientes a uma ou mais das quatro situações avaliadas. ${ }^{13}$ Esses trabalhos encontraram uma adesão maior do que os resultados encontrados no presente estudo, ressaltando-se que empregaram questionários diferentes.

Com a finalidade de aumentar a adesão à terapia imunossupressora em pacientes transplantados renais, estratégias educacionais e comportamentais mostraram resultados positivos, necessitando-se de diferentes estratégias para cada paciente. ${ }^{14} \mathrm{O}$ emprego de estratégias de intervenção tem demonstrado o aumento na adesão, necessitando-se uma compreensão do tipo de intervenção é mais efetiva. ${ }^{15}$

\section{CONSIDERAÇÕES FINAIS}

Através da utilização do BMQ, foi possível identificar as barreiras na adesão ao tratamento imunossupressor, pois quando avaliado o domínio regime, que analisou, posologia, esquemas de tratamento, horários de tomada e esquecimentos, os índices de adesão foram positivos, demonstrando que os pacientes seguem a terapia. Porém, 
ao avaliar os três domínios (regime, recordação e crenças), os índices de adesão foram baixos, pois nos domínios recordação e crença foram levados em consideração às opiniões dos pacientes sobre efeitos e suas dificuldades em tomar diariamente as medicações. Mesmo ao seguir a posologia indicada, muitos pacientes ainda permanecem em dúvida sobre eficácia, e esquema terapêutico proposto. Os resultados se confirmam nos escores do TMG, que obteve altos índices de adesão à terapia, por avaliar o uso habitual de medicamentos.

Assim, através destas avaliações é pode-se observar esquemas terapêuticos possivelmente inadequados ou incoerentes ao paciente e ainda falhas na utilização dos medicamentos. Ressalta-se a importância da utilização de questionários como ferramentas para avaliação indireta de adesão ao tratamento, promovendo com isso o uso racional de medicamentos, sendo estas ações desenvolvidas durante a atuação clínica do profissional farmacêutico.

\section{REFERÊNCIAS}

1. Bastos GM, Bregman R, Kirsztanjn MG. Doença renal crônica: frequente e grave, mas também prevenível e tratável. Rev Ass Med Bras. 2010;56:248-253.

2. Hall JE. Guyton \& Hall: tratado de fisiologia médica. 13a ed. Rio de Janeiro: Guanabara Koogan, 2017.

3. Ministério da Saúde. Secretaria de Atenção à Saúde. Portaria SAS/MS nº 712, de 13 de agosto de 2014, republicada em 14 de agosto de 2014. Protocolo clínico e diretrizes terapêuticas da imunossupressão no transplante renal.

4. Ciancio G, Burke GW, Jorge D, Rosen A, Miller J. Immunosuppressive treatment options in renal transplantation. Minerva Urol Nefrol. 2005;57:141-149.

5. Maldaner CR, Beuter M, Brondani CM, Budó MLD, Pauletto MR. Fatores que influenciam a adesão ao tratamento na doença crônica: o doente em terapia hemodialítica. Rev Gaúcha Enferm. 2008;29:647-653.

6. Santos P, Silva M. Controle terapêutico no transplante renal: estratégia de promoção do uso racional de imunossupressores. 21f. Trabalho de Conclusão de Curso- Especialização, Universidade Católica de Brasília. Brasília, 2012.

7. Ben AJ, Neumann CR, Mengue SS. Teste de Morisky-Green e Brief Medication Questionnaire para avaliar adesão a medicamentos. Rev. Saúde Pública. 2012;46:279-289.

8. Bampi SC, Leal LF, Falavigna M, Araújo LPR, Eick R, Kuhmmer R, Guterres CM, Gnatta D. Avaliação da adesão medicamentosa em pacientes em pacientes portadores de insuficiência renal crônica submetidos à hemodiálise. Rev Bras Farm Hosp Serv Saúde . 2015;6(4):12-17. 
9. Hasselmann MH, Faerstein E, Werneck GL, Chor D, Lopes CS. Associação entre circunferência abdominal e hipertensão arterial em mulheres: estudo pró-saúde. Cad Saúde Pública. 2008;24:1187-1191.

10. Mantovani FM, Mattei AT, Arthur JP, Ulbrich EM, Moreira RC. Utilização do brief medication questionnaire na adesão medicamentosa de hipertensos. Rev Enferm UFPE on line. 2015;9:84-90.

11. BEN JA. Confiabilidade e análise de desempenho de dois questionários de avaliação da adesão ao tratamento anti-hipertensivo: teste de Morisky-Green e Brief medication questionnaire. 2011. 106f. Dissertação de Mestrado- Faculdade de Medicina, Universidade Federal do Rio Grande do Sul, Porto Alegre, 2011.

12. Lins SM, Leite JL, Godoy S, Tavares JM, Rocha RG, Silva FV. Adesão de portadores de doença renal crônica em hemodiálise ao tratamento estabelecido. Acta Paul Enferm. 2018;31(1):54-60.

13. Leite RF, Silva AC, Oliveira PC, Silva LM, Pestana JM, Schirmer J, Roza BA. Mensuração da adesão aos medicamentos imunossupressores em receptores de transplante renal. Acta Paul Enferm. 2018;31(5):489-496.

14. Mathes T, Großpietsch K, Neugebauer EAM, Pieper, D. Interventions to increase adherence in patients taking immunosuppressive drugs after kidney transplantation: a systematic review of controlled trials. Syst Rev. 2017;29;6(1):236-249.

15. Zhu Y, Zhou Y, Zhang L, Zhang J, Lin J. Efficacy of interventions for adherence to the immunosuppressive therapy in kidney transplant recipients: a meta-analysis and systematic review. J Investig Med. 2017;65:1049-1056. 